\title{
Propagation of Alfvén waves in the magnetotail during substorms
}

\author{
R. L. Lysak, Y. Song, and T. W. Jones \\ School of Physics and Astronomy, University of Minnesota, 116 Church Street SE, Minneapolis, MN 55455, USA \\ Received: 30 December 2008 - Revised: 19 March 2009 - Accepted: 4 May 2009 - Published: 18 May 2009
}

\begin{abstract}
Recent observations from the THEMIS mission have focused attention on the timing of events in the magnetotail during magnetospheric substorms and other periods of geomagnetic activity. Standard models of substorms have generally emphasized convective flows as the major source of energy and momentum transport; however, Alfvén wave propagation can also be an important transport mechanism. The propagation of Alfvén waves and the related field-aligned currents are studied by means of ideal MHD simulation of the tail. Perturbations of the cross-tail current can lead to the generation of such waves, and the resulting propagation both through the tail and along the plasma sheet boundary layer can lead to enhanced transport. Implications of this wave propagation on the interpretation of results from the THEMIS mission will be discussed.
\end{abstract}

Keywords. Magnetospheric physics (Magnetotail; MHD waves and instabilities; Storms and substorms)

\section{Introduction}

The propagation of MHD waves and wave pulses in the magnetotail represents an important mode of energy and momentum transport between this region and the inner magnetosphere. On slow time scales, convection of the magnetospheric plasma is the major mechanism to transport mass, energy and magnetic flux throughout the bulk of the magnetosphere. On the other hand, during substorms when there are rapid changes in the magnetospheric stresses, wave propagation can be a competitive factor in transporting energy and momentum (Song, 1998). Ultra-Low-Frequency (ULF) waves play a number of roles in the overall picture of tail dynamics. Such waves are responsible for the communication of shear and compressional stresses throughout the magne-

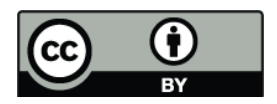

Correspondence to: R. L. Lysak (bob@aurora.space.umn.edu) tosphere, and in the tail in particular. Shear Alfvén waves mediate changes in the field-aligned currents that are generated during substorms. The rapid enhancement in the nightside field-aligned currents that constitute the substorm current wedge is carried by these Alfvén waves. Compressional fast mode waves can transmit changes in the total pressure between different regions of the tail. For example, the dipolarization that occurs during the substorm could be viewed as a rarefactive wave propagating from the inner tail to the mid-tail region, or conversely as a compressional wave propagating earthward from the mid-tail. These waves often occur in isolated pulses or wave packets, rather than continuous sinusoidal waves. The dynamics of these wave packets is an important consideration for the evolution of the magnetosphere, especially during substorms (e.g., Song and Lysak, 1994, 2000, 2001). In this paper, we will use "waves" and "wave packets" interchangeably, since the propagation characteristics of waves and wave packets are similar.

The importance of wave propagation to energy transport between the tail and the auroral zone has been emphasized by observations at the plasma sheet boundary layer by Polar, Cluster, and FAST (Wygant et al., 2000, 2002; Keiling et al., 2000, 2001, 2002, 2003, 2005; Angelopoulos et al., 2002; Nakamura et al., 2004; Dombeck et al., 2005). These observations show that the Poynting flux carried by shear Alfvén waves can be the dominant energy flow along auroral field lines, especially during substorm times. Angelopoulos et al. (2002) have considered examples when the Geotail and Polar satellites were conjugate to one another, and determined that a large fraction of the Poynting flux observed at larger distances by Geotail was dissipated before reaching Polar altitudes. In addition, they pointed out that the energy flux associated with bursty bulk flows may be converted to Poynting flux associated with shear Alfvén waves. Similar wave activity has been seen from Cluster by Nakamura et al. (2004) who determined with direct multi-point measurements that these waves had wavelengths perpendicular to the magnetic field as small as the electron inertial

Published by Copernicus Publications on behalf of the European Geosciences Union. 
length. These results verified the indirect result of Wygant et al. (2002) who used the local kinetic dispersion relation (Lysak and Lotko, 1996; Lysak, 1998) to estimate the perpendicular wavelength from the ratio between the wave electric and magnetic fields. In addition, Wygant et al. (2002) have suggested that these Alfvén waves may have a direct effect on particle acceleration, since they observe accelerated field-aligned electrons, sometimes counter-streaming, in the presence of short-perpendicular wavelength Alfvén waves. This electron energization is consistent with the parallel potentials expected in these waves from the kinetic Alfvén wave dispersion relation. Furthermore, Mende et al. (2003) have shown from FAST and IMAGE observations that the auroral arc that brightens at substorm onset is due to the Alfvénic acceleration of electrons rather than a quasi-static inverted-V electron population.

Previous theories of field-aligned current generation (e.g., Hasegawa and Sato, 1979; Sato and Iijima, 1979; Vasyliunas, 1984) are based on a quasi-static force balance and current continuity, and so cannot describe the highly dynamical situation occurring at substorm onset. Since the shear Alfvén wave carries changes in the field-aligned current, a dynamical theory of field-aligned current generation must also involve the generation of shear Alfvén waves. In an ideal MHD situation, Ampere's Law and Faraday's Law can be combined with the ideal Ohm's Law to show that currents are generated when there is a gradient in the field-aligned vorticity along magnetic field lines (Song and Lysak, 1997, 2000). In the magnetotail, this situation can arise during three-dimensional, time-dependent reconnection. Such reconnection will produce a plasma flow localized in space and time, both Earthward and anti-Earthward from the reconnection site. Since this flow is localized in the azimuthal direction, there will be a field-aligned vorticity produced that is southward on the dawn side and northward on the dusk side. This will produce a field-aligned current toward the Earth on the dawn side and away from the Earth on the dusk side (e.g., Birn and Hesse, 1991, 2000). Currents produced by such localized flows have been observed by Cluster (e.g., Volwerk et al., 2004, 2007), and were associated with the Kelvin-Helmholtz instability.

Another proposed current generation mechanism occurs during the so-called Hall reconnection including two-fluid effects (e.g., Sonnerup, 1979; Birn and Hesse, 2001; Shay et al., 2001). In this case the demagnetization of ions in a thin current layer causes an overshoot, leading to a perpendicular current away from the reconnection site. On the other hand, the confinement of electrons to a narrower current layer leads to an electric field pointed toward the reconnection site. Thus, in this situation, $\boldsymbol{j} \cdot \boldsymbol{E}<0$, constituting a generator. The resulting outgoing Alfvén waves produce a quadrupolar signature in the out-of-plane component of the magnetic field that has recently been observed on satellites (e.g., Øieroset et al., 2001; Mozer et al., 2002).
A final mechanism that will be considered for field-aligned current generation is linear mode conversion at the plasma sheet boundary layer (e.g., Hasegawa, 1976; Goertz and Smith, 1989; Harrold et al., 1990; Allan and Wright, 1998, 2000; and Wright and Allan, 2008). If fast mode waves are produced in the plasma sheet, they will propagate nearly isotropically through the sheet, and will quickly reach the boundary layer. Due to the strong gradient in the Alfvén speed at this layer, the fast mode can convert into the shear Alfvén mode, similar to the effect that occurs in the excitation of field line resonances. These Alfvén waves will then propagate along the field line, where they can reach the Earth. Such waves have been observed at the boundary layer by Polar (Wygant et al., 2000; Keiling et al., 2003). They may possibly be associated with Poleward Boundary Instensifications frequently seen during the later stages of substorms (Lyons et al., 2002).

The purpose of this paper is to investigate the propagation of Alfvén waves in the magnetotail during substorms by means of a three-dimensional ideal MHD code (Ryu et al., 1998). This code treats purely ideal MHD, and thus cannot describe the physics of reconnection or kinetic instabilities; however, it does have very low numerical dissipation so that the wave propagation can be accurately treated. The next section will briefly describe this code. The following two sections will describe results from two runs of the code. The first considers a region farther back in the tail in which the current sheet can be modeled as a Harris-type equilibrium with a finite plasma sheet including narrow boundary layers and the transition to the lobes. This model can be used to study mode conversion at the plasma sheet boundary layer. The second case will consider a region closer to the Earth where a finite $B_{z}$ component of the background magnetic field is present, which will allow a study of field-aligned current generation during current disruption and the possible propagation of rarefactive waves tailward. This model uses a Lembege-Pellat approximate tail equilibrium as the starting point. In each of these runs, the current sheet will be perturbed by reducing the current strength locally. The results will be discussed in the final section.

\section{Numerical model}

Our main computational tool to examine these questions is an existing three-dimensional MHD model that has been developed by the University of Minnesota Astronomy Department (Ryu and Jones, 1995; Ryu et al., 1995). This code is based on a total variance diminishing (TVD) conservative scheme that has proven to have very low numerical dissipation (Ryu et al., 1995). This code has been used to study the MHD Kelvin-Helmholtz instability in two dimensions (Frank et al., 1996; Jones et al., 1997) as well as in three dimensions (Jones et al., 1999; Ryu et al., 2000). The threedimensional code has been applied to several challenging 
astrophysical problems, including the Parker instability (Kim et al., 1998, 2001), and supersonic MHD jets (O'Neill et al., 2005). This code has been applied to magnetospheric situations such as the Kelvin-Helmholtz instability at the magnetopause, including the effects of oblique magnetic fields (Keller and Lysak, 1999), the generation of field-aligned current in three dimensions (Keller et al., 2000), and the excitation of magnetospheric waveguide modes (Keller and Lysak, 2001). In addition, we have used this code to study mode conversion at the plasma sheet boundary layer (Lysak and Song, 2004).

This code is well suited to studying wave propagation in the tail. In this code each cell interface is treated as a Riemann problem considering the 7 MHD wave characteristics (corresponding to propagation at the convection speed $u$ plus the wave speeds $u \pm c_{i}$, where $c_{i}, i=1,2,3$ are the fast, intermediate and slow MHD wave speeds). The order in which the interfaces in the three dimensions are computed is permuted to preserve second-order accuracy. Outflow boundary conditions are implemented by considering only the outbound wave characteristics on the boundary. The code was tested by introducing fast, shear and slow waves propagating obliquely across the grid, and effective Reynolds numbers the order of $10^{4}$ were achieved in a grid with 256 cells in each direction (Ryu et al., 1995). In general, the directional splitting used in the code would not preserve $\nabla \cdot \boldsymbol{B}=0$. Maintenance of a divergence-free magnetic field is accomplished in this code by a non-directionally split solution of Faraday's Law, calculating the magnetic flux transport across each cell boundary by finding the electric field along the cell edges and computing the emf around each cell face (Dai and Woodward, 1998; Ryu et al., 1998).

The eigenmode structure of MHD waves in the magnetotail geometry has been considered for many years (e.g., Patel, 1968; Siscoe, 1969; McKenzie, 1970; Hopcroft and Smith, 1985; Seboldt, 1990; Rickard and Wright, 1994; Allan and Wright, 1998, 2000; Wright and Allan, 2008; Lee and Hau, 2008). These studies show that the magnetotail can constitute a waveguide for compressional mode waves. Finite difference modeling of these waveguide modes has been done by Allan and Wright $(1998,2000)$ and Wright and Allan (2008) using a linear, cold plasma model. The model presented here improves on these models by considering a fully nonlinear, warm plasma MHD description of the tail. A nonlinear resistive MHD code has been developed by Birn and co-workers (Birn and Hesse, 1991, 1996, 2000; Birn et al., 1996). Our model is quite similar to this work; however, we treat an ideal MHD model that has lower dissipation than these models, allowing a good description of the wave propagation. On the other hand, without resistive terms our model cannot directly model the reconnection region that was considered by Birn's models.

For the runs presented below, the box size is $10 R_{E} \times 6 R_{E} \times 6 R_{E}$ (with the longer side being in the GSM $x$ direction) with a spatial resolution of $200 \mathrm{~km}$, giving

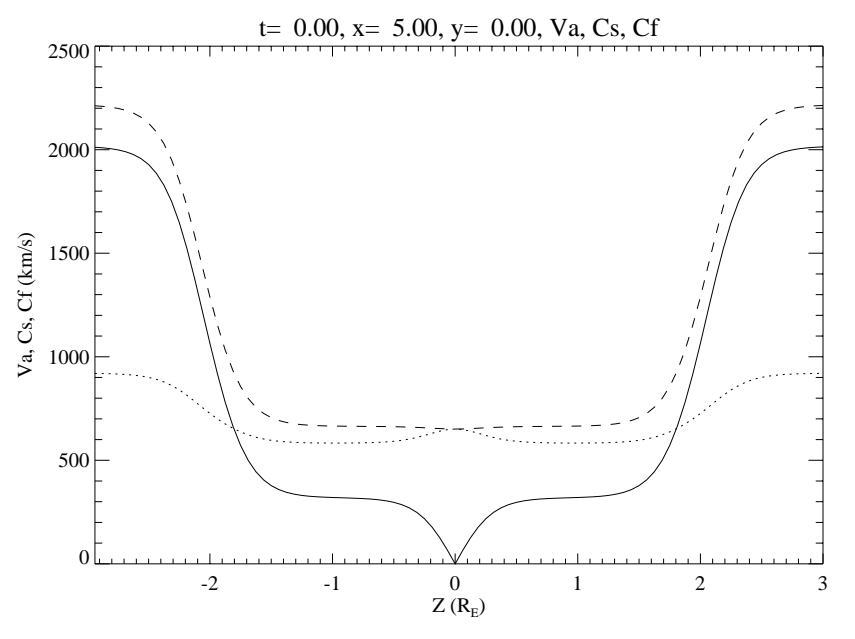

Fig. 1. Profiles of the Alfvén speed (solid), sound speed (dotted), and fast mode speed (dashed) for the profiles used in the Harris sheet runs.

$320 \times 192 \times 192$ cells. Outflow conditions are assumed at each boundary, and the runs are done as an initial value problem, where the initial equilibrium is perturbed by a local reduction in the cross-tail current. This reduction mimics the effects of non-ideal MHD processes within the context of our ideal MHD model. This perturbation is modeled by a three-dimensional Gaussian in the vector potential, which models an obstacle (such as localized resistivity) that current must flow around (Song and Lysak, 1989; see also Fig. 2b in Lysak and Song, 1990). It should be noted that such a perturbation could either be the result of localized, three-dimensional reconnection at the current sheet or the result of a localized resistivity due to a kinetic current instability.

\section{Harris sheet profile including boundary layer}

A first attempt to model wave propagation in the tail was attempted using a Harris sheet profile including the plasma lobe and thus gradients at the boundary layer. First, the magnetotail current sheet is modeled by a hyberbolic tangent profile that reverses the magnetic field from a value of $-15 \mathrm{nT}$ to $15 \mathrm{nT}$ over a sheet thickness of $0.3 R_{E}$. The density in the asymptotic region is $1 \mathrm{~cm}^{-3}$ and $\beta=4$, with pressure balance assumed throughout. The boundary layers are modeled by hyperbolic tangents centered at $z_{B L}= \pm 1.8 R_{E}$ with a thickness of $0.3 R_{E}$ that increase the magnitude of the magnetic field to $30 \mathrm{nT}$ and decrease the density to $0.1 \mathrm{~cm}^{-3}$. Again the pressure is in balance with the magnetic field pressure, leading to $\beta=0.25$ in the lobe regions. Thus, the total magnetic field can be written as

$B_{x}(z)=B_{P S} \tanh \frac{z}{\Delta z}+\frac{B_{L}}{2}\left(\tanh \frac{z+z_{B L}}{\Delta z}+\tanh \frac{z-z_{B L}}{\Delta z}\right)(1)$ 

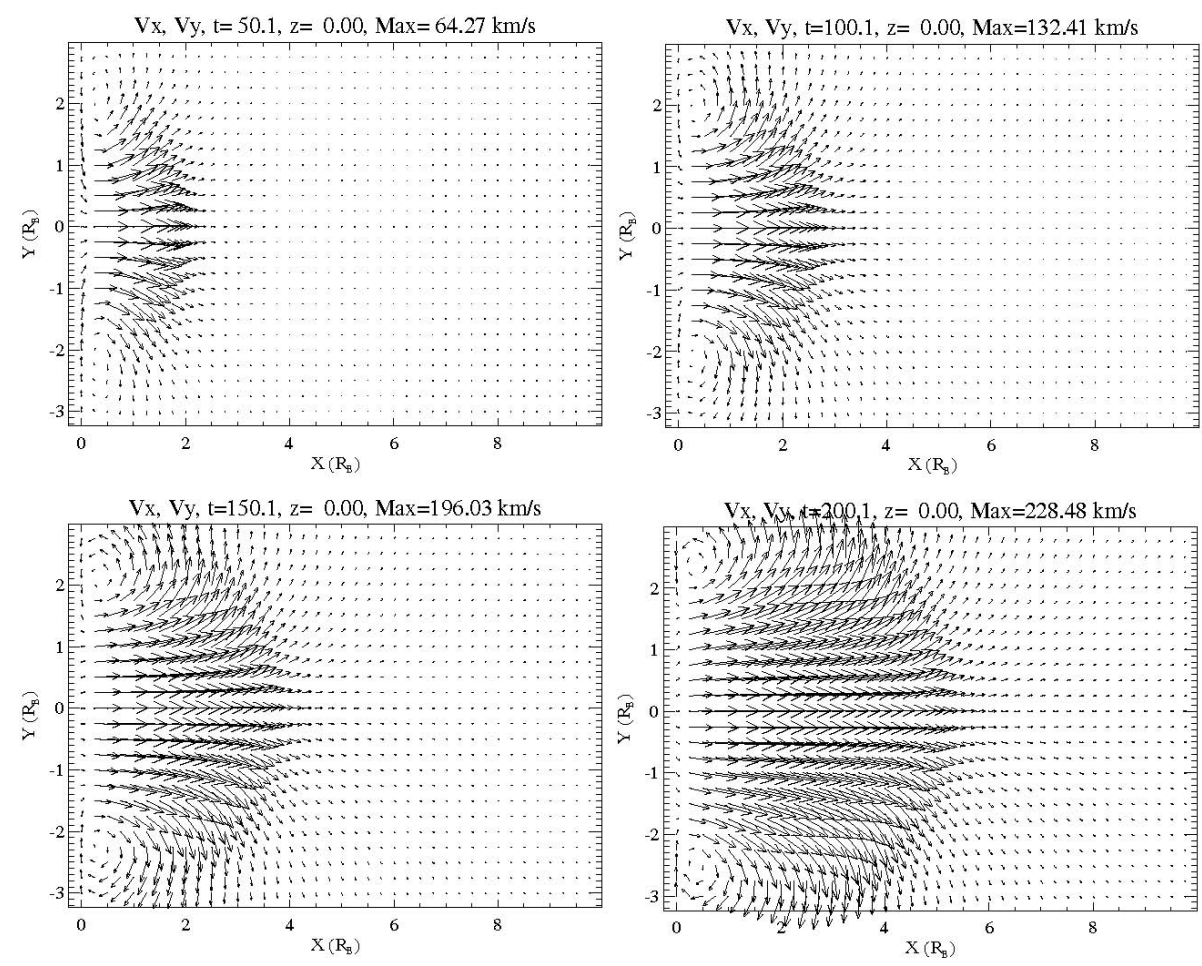

Fig. 2. Velocity vectors in the $z=0$ plane at $50,100,150$, and $200 \mathrm{~s}$ into the run.

where $B_{P S}=15 \mathrm{nT}, B_{L}=30 \mathrm{nT}$ and $\Delta z=0.3 R_{E}$. Figure 1 shows the resulting profile of the wave speeds. In this figure, the solid line denotes that Alfvén speed, the dotted line the sound speed, and the dashed line the fast mode speed. Note that $\gamma=5 / 3$ is assumed.

In this run, the reduction of the cross-tail current is modeled by a Gaussian perturbation in the vector potential that has the form:

$A_{y}(\boldsymbol{x})=A_{0} e^{-\left(x^{2}+z^{2}\right) / \Delta_{\perp}^{2}} e^{-y^{2} / \Delta y^{2}}$

where $\Delta_{\perp}=0.3 R_{E}$ and $\Delta y=1.0 R_{E}$. This perturbation was introduced at the left side $(x=0)$ of the simulation volume. The magnetic field produced by this perturbation takes the form

$\boldsymbol{B}(\boldsymbol{x})=\frac{2 A_{y}}{\Delta_{\perp}^{2}}(z \hat{\boldsymbol{x}}-x \hat{z})$

This magnetic perturbation can be described as a toroidal perturbation that is associated with a poloidal current. This perturbation reduces the cross-tail current in the center of the perturbation, diverting the current around the perturbation so that $\nabla \cdot \boldsymbol{j}=0$ is preserved. This configuration results in an unbalanced $\boldsymbol{j} \times \boldsymbol{B}$ force that will accelerate plasma to the right (i.e., Earthward in this case). Figure 2 shows the flow vectors in the $z=0$ plane at 50,100,150 and $200 \mathrm{~s}$ into the run. The acceleration of the plasma flow by the unbalanced forces can be clearly seen. Note in particular that the maximum velocity increases by about $65 \mathrm{~km} / \mathrm{s}$ in each panel, although by $t=200 \mathrm{~s}$ the acceleration has slowed as the field lines relax. It can also be seen that due to the finite extent of the accelerated plasma in the y-direction, the flow diverts and forms vortices at the endpoints, similar to previous simulations (e.g., Birn and Hesse, 1991).

Figure 3 shows the total pressure $\left(p+B^{2} / 2 \mu_{0}\right)$ in the $y=0$ plane at the same times as in Fig. 2. Perturbations in the total pressure are indicative of fast mode pulses. Comparing these figures, one can see that there is a peak in the pressure at the leading edge of the flow pattern. In addition to this expected pattern, it can be seen that there is a leading front of enhanced pressure that runs ahead of the flow pattern, representing a fast mode perturbation. Note that this perturbation expands to fill the plasma sheet, as it should since the fast mode propagates nearly isotropically. In particular, this perturbation reaches the plasma sheet boundary layer, where it can mode convert to an Alfvén wave propagating along the magnetic field lines. In this run, the initial pressure was $0.425 \mathrm{nPa}$ everywhere in the system, so the fluctuations in the pressure are only about $\pm 10 \%$. Thus, this fast wave pulse is rather weak and might not stand out clearly in observations.

The Earthward component of the current (essentially the field-aligned current) is illustrated in Fig. 4, which plots the current at times of 70, 80, 90, and $100 \mathrm{~s}$. These field-aligned currents are signatures of Alfvén mode perturbations. This figure is a cut offset from the center of the simulation, at $y=1.8 R_{E}$, since the current has a node at $y=0$. The black lines in this figure are the points where $j_{x}=0$. Strong currents 

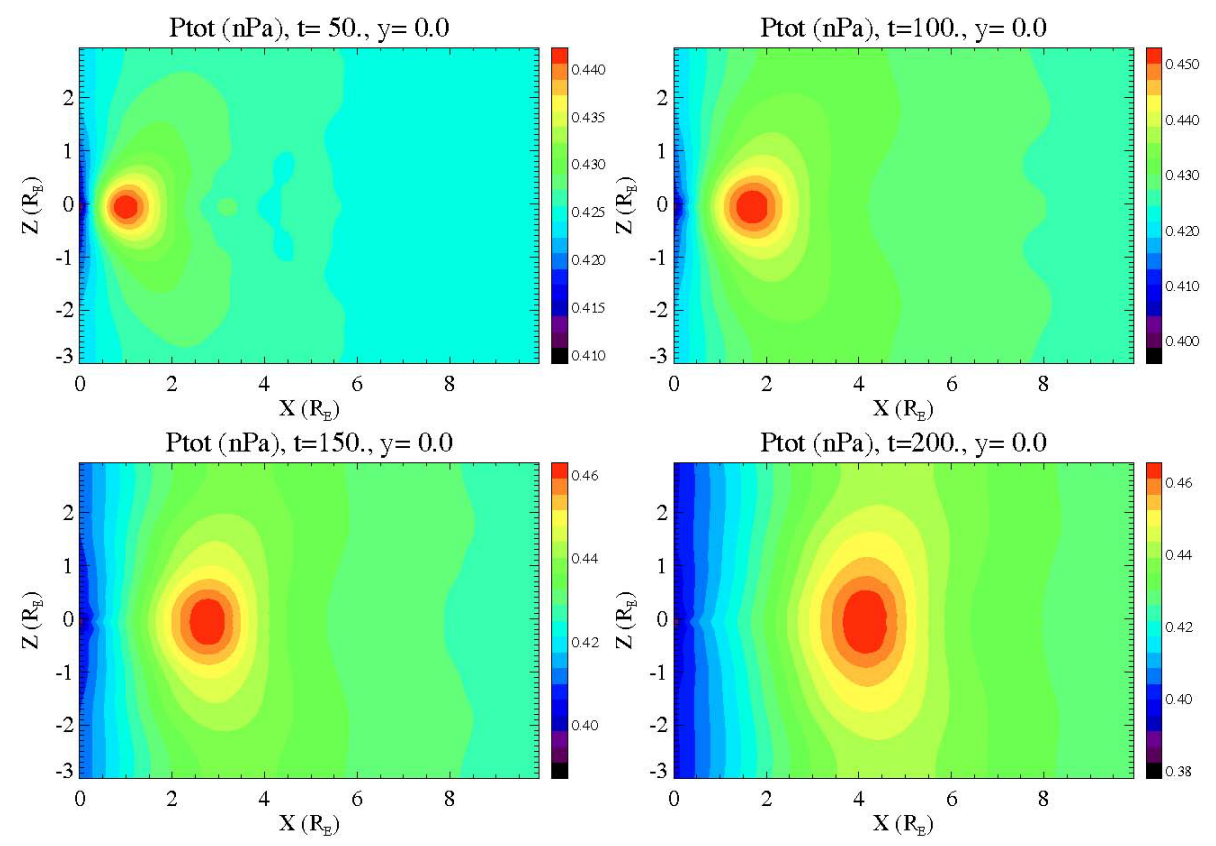

Fig. 3. Contours of the total pressure $\left(p+B^{2} / 2 \mu_{0}\right)$ in the $y=0$ plane at the same times as in Fig. 2.
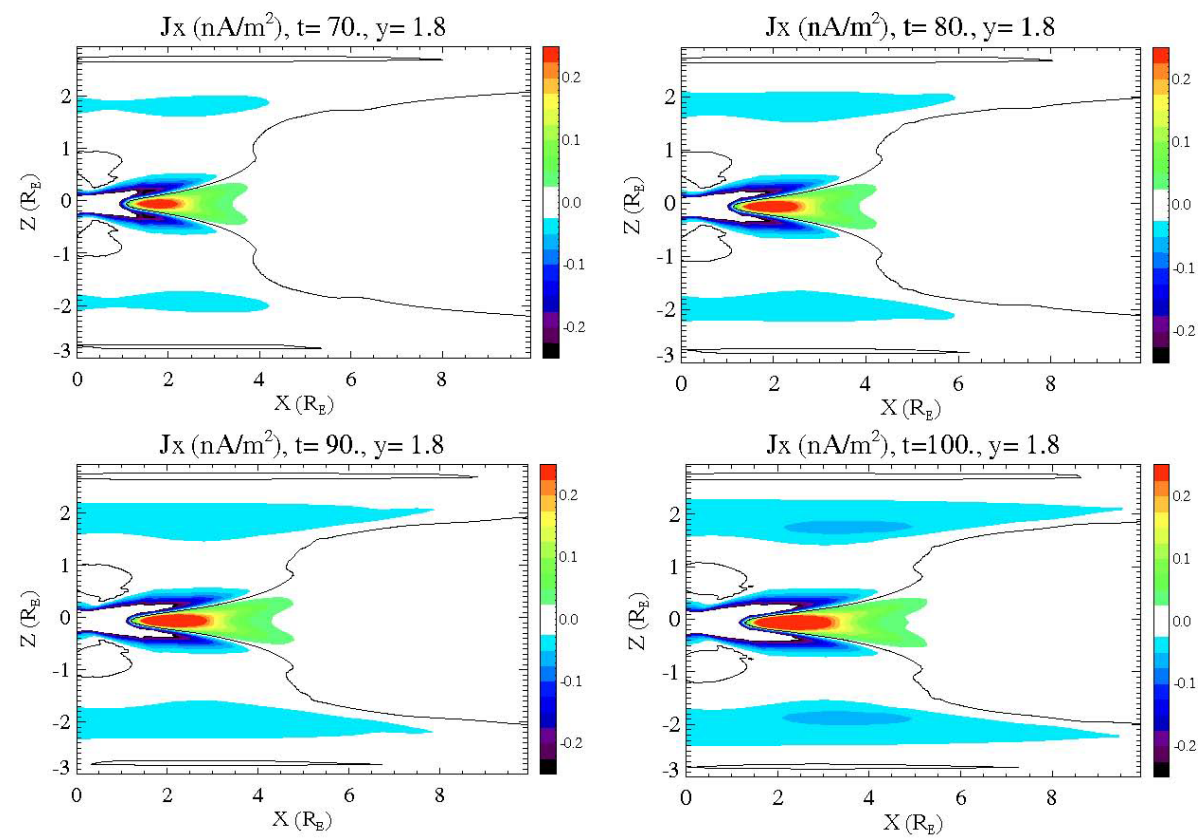

Fig. 4. Contours of the $j_{x}$ current at 70, 80, 90, and $100 \mathrm{~s}$ into the run. Blue and black colors are negative (tailward) currents and green and red are positive (Earthward currents). The black contours give the zero current level.

with both signs are found near the central current sheet in this figure. These are the currents directly related to the flow vortices shown in Fig. 2. These currents correspond to those seen, for example, in Birn et al. (1996). For strong flows, these flow shears can be unstable to the Kelvin-Helmholtz instability, as observed by Cluster (Volwerk et al., 2007); how- ever, in this case, the flows are weaker than those in the Cluster observations. In addition to these currents, however, are currents at $z \sim \pm 1.8 R_{E}$, at the location of the plasma sheet boundary layer. In contrast to the earlier figures, here there is a time difference of only $10 \mathrm{~s}$ between the panels in order to show the rapid propagation of the Alfvén wave carrying these 

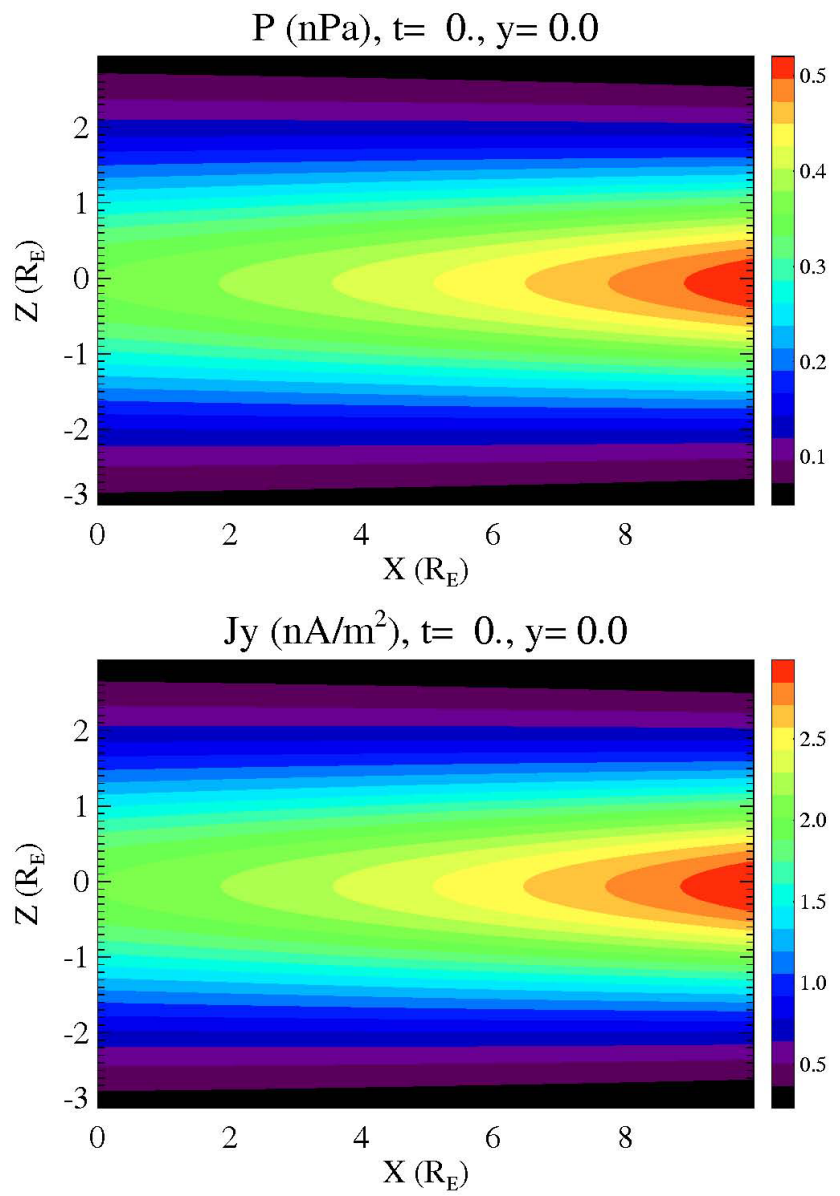

Fig. 5. Contours of pressure (top) and cross-tail current (bottom) for the equilibrium model described in Eqs. (2) and (3). Note that the pressure contours are equivalent to magnetic field lines.

currents at the boundary layer. The current signature travels about $6 R_{E}$ in $30 \mathrm{~s}$, corresponding to a velocity of $1280 \mathrm{~km} / \mathrm{s}$, comparable to the Alfvén speed in the boundary (see Fig. 1). It is important to note that there is no significant plasma flow in the boundary layer at these times, and so the excitation of these Alfvén waves is a mode conversion effect as described above.

Thus, this run illustrates the ability of a generation of Alfvén waves at the plasma sheet boundary layer by mode conversion, even though the original reduction of the current (which could be, but is not necessarily, due to reconnection) is confined to a current sheet embedded within the plasma sheet. The wave propagation speed at the boundary layer is much faster than the wave speed in the center of the plasma sheet, and so a more rapid communication of the state of the tail to the inner magnetosphere is possible. Such rapid wave propagation at the boundary layer may be responsible for the so-called poleward boundary intensifications (Lyons et al., 2002).

\section{Tail model with finite $B_{z}$}

While the Harris sheet model above is instructive, it is not very realistic, particularly closer to the Earth where a finite $B_{z}$ component to the field is likely. In such a case, there are a number of quasi-equilibrium solutions (see, e.g., Lui, 2004) based on the assumption that variations in the downtail $(x)$ direction are weaker than those in the north-south $(z)$ direction. Here, we will adopt the model of Lembége and Pellat (1982), in which the vector potential takes the form:

$A_{y}=h(x)-\lambda B_{0} \ln \cosh \left[\frac{z}{\lambda} \exp \left(\frac{h(x)}{\lambda B_{0}}\right)\right]$

where $h(x)$ is a slowly varying function. Note that this model reduces to the Harris sheet in the case where $h$ is 0 . For the model presented here we will take $h(x)=B_{0} \varepsilon x e^{\varepsilon x / \lambda}$. In this model, the magnetic field at $z=0$ is just $B_{z}=d h / d x$ and the pressure is given by

$p(x, z)=\frac{B_{0}^{2}}{2 \mu_{0}} e^{2 A_{y} / \lambda B_{0}}$

Figure 5 shows (a) the contours of pressure for this model (which are the same as the magnetic field lines) and (b) the magnitude of the cross-tail current for this case. Here the parameters are $B_{0}=30 \mathrm{nT}, \varepsilon=0.03$, and $\lambda=1.8 R_{E}$, so that the zcomponent of the magnetic field at $z=0$ ranges from $0.9 \mathrm{nT}$ to $1.24 \mathrm{nT}$ as $x$ increases from 0 to $10 R_{E}$. The tail-like geometry can be clearly seen as well as the fact that the cross-tail current increases as one moves toward the Earth.

This run is initiated with a perturbation that reduces the cross tail current at the center of the system from its initial value of $2.4 \mathrm{nA} / \mathrm{m}^{2}$ to $0.5 \mathrm{nA} / \mathrm{m}^{2}$. The state of the system after $100 \mathrm{~s}$ is shown in Fig. 6. The first panel of this figure shows the velocity vectors in the $z=0$ plane. A four-fold vortex structure is clearly seen. This structure is formed since the $B_{z}$ component associated with the current perturbation gives a $\boldsymbol{j} \times \boldsymbol{B}$ force in the $\mathrm{x}$-direction that drives plasma away from the region of the perturbation. The diverging flow of plasma away from this region leads to a reduction in the total pressure, providing a force that accelerates the plasma in the $y$-direction to fill the low pressure spot. This local reduction in the pressure sends a fast mode signal through the tail, as shown in panel (b) of the figure. Again, this fast mode wave is not very strong, having a pressure variation of $\pm 0.1 \mathrm{nPa}$.

The vortical motion of the plasma leads to a twisting of field lines, and thus to the generation of field-aligned current. This current is shown in Fig. 6c, which shows the $x$ component of the current on the $y=1$ plane (note that this is only approximately the field-aligned current). This plane is on the pre-midnight side of the magnetosphere, and so the negative currents in the direction of the Earth indicate upward currents in the ionosphere, in the proper sense for Region 1 currents, or alternatively, the pre-midnight leg of the substorm current wedge. Similar current structures have been seen in simulations by Birn and Hesse (2000). Finally, Fig. 6d shows the 

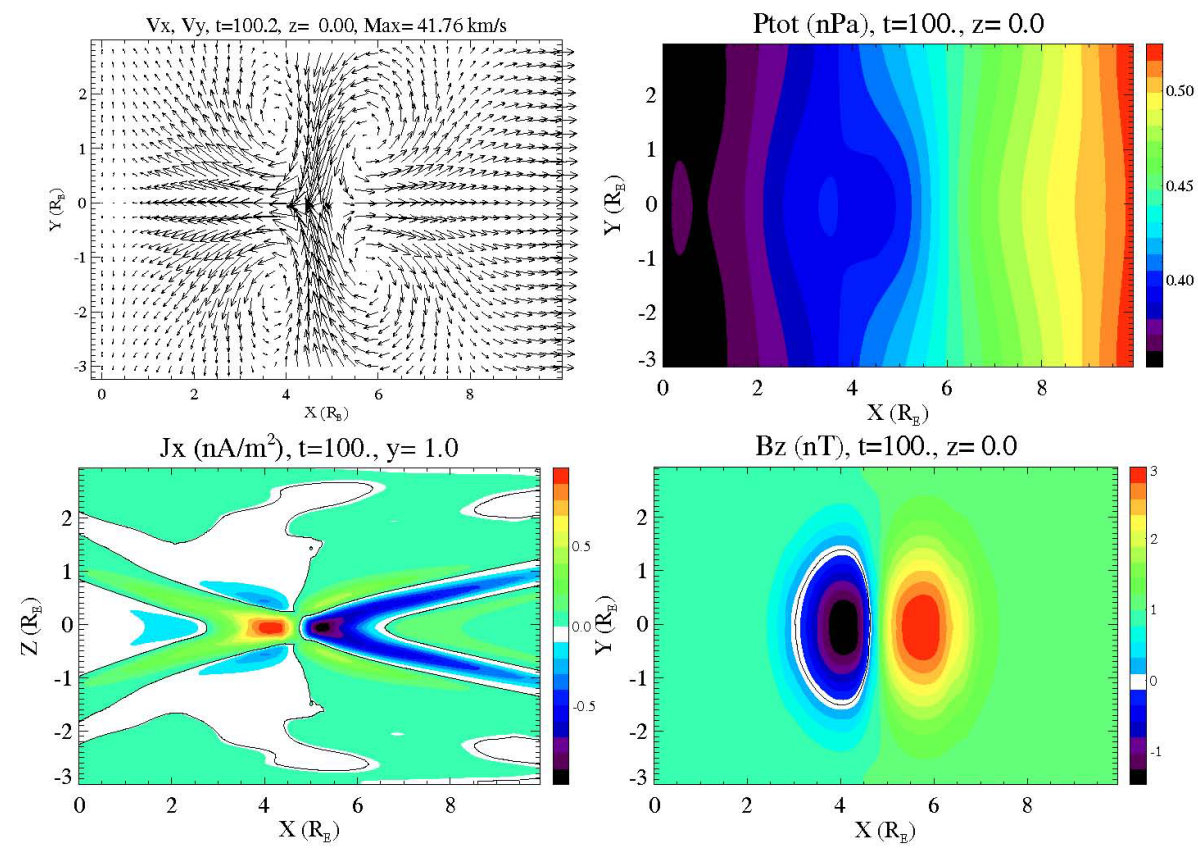

Fig. 6. Fields at $100 \mathrm{~s}$ into the run based on the equilibrium model in Fig. 5. (a) Top left: velocity vectors in the $z=0$ plane; (b) Top right: Total pressure contours for the $z=0$ plane; (c) Bottom left: Contours of the Earthward current at the $y=1$ plane; and (d) Bottom right: Contours of the $B_{z}$ magnetic field.

$B_{z}$ component of the magnetic field, indicating that a region of negative $B_{z}$ occurs just tailward of the perturbation. Since the perturbation is localized in the y-direction, this localized region of negative $B_{z}$ does not necessarily involve the formation of a large scale plasmoid heading tailward in this case.

A final point to be made from this run is illustrated in Fig. 7, which shows the $B_{y}$ component of the magnetic field on the $y=1$ plane at the same time as the fields shown in Fig. 6. It can be seen that a quadrupolar pattern in $B_{y}$ occurs, consistent with the currents shown in Fig. 6c. It is worth noting that such a pattern is often invokes as the signature of "Hall reconnection;" however, this run is calculated using ideal MHD without the Hall term. It is probably more accurate to describe such a pattern as the signature of localized field-aligned current generation in the tail. Certainly, such current generation could be the result of the charge separation that occurs in thin current sheets due to the breakdown of the ion frozen-in condition (i.e., the Hall term), but this is not the only way in which such currents can be generated.

There are at least two distinguishing characteristics between the pattern shown in Fig. 7 and that produced by twofluid effects in reconnection. The first is the scale size, which is much larger in Fig. 7 than the ion inertial length. Hall reconnection produces $B_{y}$ signatures that are the order of a few times the ion inertial length, as is seen in both data and simulation (e.g., Phan et al., 2007). Larger scale regions of this out-of-plane magnetic field would indicate alternative current generation scenarios. The second difference is

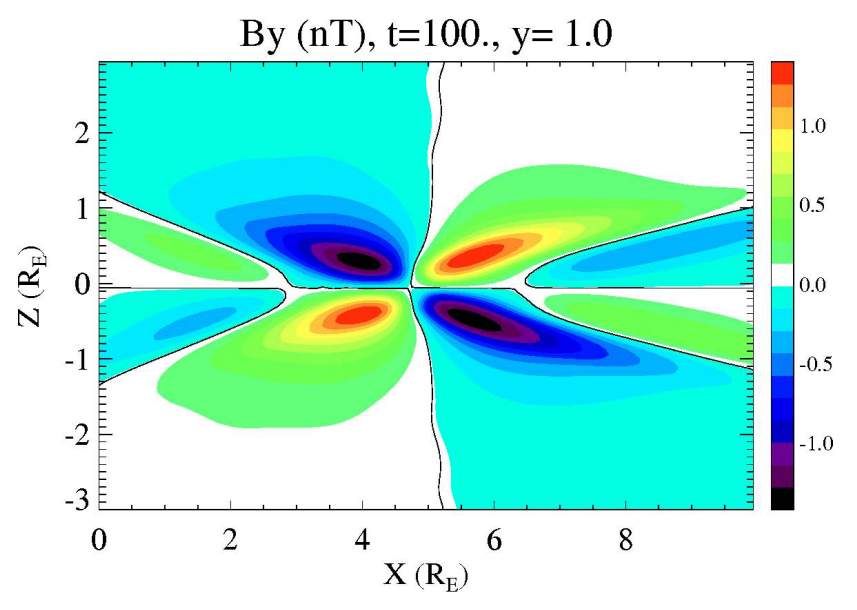

Fig. 7. Contours of the $B_{y}$ component in the $y=1$ plane. Note the quadrupolar pattern, similar to that found in the Hall reconnection picture. However, this differs since the polarity of the field is reversed for negative values of $y$.

that the Hall reconnection picture is two-dimensional, and so the same pattern is seen independent of the y-coordinate. In the case of a localized three-dimensional current generation process, the pattern reverses depending on whether the observations are duskward and dawnward of the reconnection region. For example, the perturbations at $y=-1$ in our simulations (not shown) has exactly opposite sign to that given in Fig. 7. Observations at points separated in $y$ might then be 
able to see such differences. While the Cluster spacecraft are relatively closely spaced, observations from THEMIS might shed light on this question.

\section{Conclusions}

These results have illustrated the relationship of Alfvén waves and field-aligned currents to perturbations in the tail current sheet. From the point of view of this work, we make no distinction between magnetic reconnection and current disruption processes: both processes lead to the localized reduction of the cross-tail current. It is important to recognize that this localized reduction might not necessarily lead to field-aligned current generation, as is implied by the standard picture of the substorm current wedge. In principle, this current may just be diverted around the region of reduction. However, in the dynamical situation shown here, the plasma flows caused by the force imbalance produced by the current reduction act as a generator, twisting field lines (e.g., Song, 1998).

Since the localized current perturbations produce a localized enhancement of the flow, the plasma is compressed at the leading edge of the flow and reduced in the region of the perturbation. The resulting pressure perturbation leads to fast mode fronts that can propagate nearly isotropically through the plasma sheet. Since these waves are not confined to a single field line, they generally do not grow to large amplitudes and so would not produce an obvious signal in spacecraft data. However, when these fast mode waves encounter the plasma sheet boundary layer, they can mode convert into shear Alfvén waves that propagate along the boundary layer field lines. These waves could propagate either toward or away from the Earth; those that propagate toward the Earth may lead to particle acceleration (e.g., Wygant et al., 2002) and could produce poleward boundary intensifications (Lyons et al., 2002) in the aurora. It should be noted that in the present simulation, which is an initial value simulation without any continuous driving, the electric fields produced are the order of $1 \mathrm{mV} / \mathrm{m}$, less than those observed by Wygant et al. (2000) even when enhancements due to magnetic field mapping are taken into account. Further driving of the system and enhancement of the field magnitudes due to continuous phase mixing would be required to explain the observed field magnitudes.

As a final point, the localized generation of field-aligned current generally leads to the formation of a $y$ component of the magnetic field. This is often called the "Hall magnetic field." However, the Hall effect is only one way to generate such a signature, and this field should more accurately be described as an indicator of field-aligned current generation. Observations of the scale size of the region, such as are often found from Cluster, are needed to confirm that such structures scale with the ion inertial length. Indeed, this whole structure is sometimes referred to as the "Hall cur- rent system" which is misleading since most of the observed currents are field-aligned. Only the closure current near the reconnection region can accurately be described as the Hall current. Rather than being the signature of whistler mode waves, which are often associated with the Hall term, these fields are usually better described as shear Alfvén waves that carry energy away from the reconnection region and lead to field-aligned currents that can propagate to low altitudes and accelerate auroral particles.

In conclusion, the results presented here have illustrated the role of MHD wave propagation in the tail in two different situations. In the Harris sheet model including the boundary layers, the simulations show that fast mode waves are generated that can mode convert to shear Alfvén waves at the boundary layer. The second model based on an equilibrium model including finite $B_{z}$ in the initial state has shown how a localized current disruption can generate field-aligned currents due to the force imbalance that generates vortical flows in the plasma sheet. Such a scenario also produced fast mode waves that propagate tailward and a quadrupolar $B_{y}$ signature that is generally indicative of localized field-aligned current generation, either due to Hall effects or simply the threedimensional structure of the currents. Future work will couple this tail model to a model of the inner magnetosphere so that these current perturbations can be connected to the auroral processes that are an essential feature of substorm dynamics.

Acknowledgements. The work was supported in part by NASA grants NNG05GM38G and NNX08AM31G. Computing resources were provided by the Minnesota Supercomputer Institute.

Topical Editor I. A. Daglis thanks J. Birn and K.-H. Glassmeier for their help in evaluating this paper.

\section{References}

Allan, W. and Wright, A. N.: Hydromagnetic wave propagation and coupling in a magnetotail waveguide, J. Geophys. Res., 103, 2359-2368, 1998.

Allan, W. and Wright, A. N.: Magnetotail waveguide: Fast and Alfvén waves in the plasma sheet boundary layer and lobe, J. Geophys. Res., 105, 317-328, 2000.

Angelopoulos, V., Chapman, J. A., Mozer, F. S., Scudder, J. D., Russell, C. T., Tsuruda, K., Mukai, T., Hughes, T. J. and Yumoto, K.: Plasma sheet electromagnetic power generation and its dissipation along auroral field lines, J. Geophys. Res., 107(A8), 1181, doi:10.1029/2001JA900136, 2002.

Birn, J. and Hesse, M.: The substorm current wedge and fieldaligned currents in MHD simulations of magnetotail reconnection, J. Geophys. Res., 96, 1611-1618, 1991.

Birn, J. and Hesse, M.: Details of current disruption and diversion in simulations of magnetotail dynamics, J. Geophys. Res., 101, 15345-15358, 1996.

Birn, J. and Hesse, M.: The current disruption myth, in: Magnetospheric Current Systems, edited by: Ohtani, S.-I., Fujii, R., Hesse, M., and Lysak, R. L., AGU Monograph 118, American Geophysical Union, Washington, D.C., p. 285, 2000. 
Birn, J. and Hesse, M.: Geospace Environment Modeling (GEM) magnetic reconnection challenge: Resistive tearing, anisotropic pressure, and Hall effects, J. Geophys. Res., 106, 3737-3750, 2001.

Birn, J., Hesse, M., and Schindler, K.: MHD simulations of magnetotail dynamics, J. Geophys. Res., 101, 12939-12954, 1996.

Dai, W. and Woodward, P. R.: On the divergence-free condition and conservation laws in numerical simulations for supersonic magnetohydrodynamical flows, Astrophys. J., 494, 317-335, 1998.

Dombeck, J., Cattell, C., Wygant, J. R., Keiling, A., and Scudder, J.: Alfvén waves and Poynting flux observed simultaneously by Polar and FAST in the plasma sheet boundary layer, J. Geophys. Res., 110, A12S90, doi:10.1029/2005JA011269, 2005.

Frank, A., Jones, T. W., Gaalaas, J. B., and Ryu, D.: The MHD Kelvin Helmholtz instability: A two dimensional numerical study, Astrophys. J., 460, 777-793, 1996.

Goertz, C. K. and Smith. R. A.: The thermal catastrophe model of substorms, J. Geophys. Res., 94, 6581-6596, 1989.

Harrold, B. G., Goertz, C. K., Smith, R. A., and Hansen, P. J.: Resonant Alfvén Wave Heating of the Plasma Sheet Boundary Layer, J. Geophys. Res., 95(A9), 15039-15046, 1990.

Hasegawa, A.: Particle acceleration by MHD surface wave and formation of aurora, J. Geophys. Res., 81, 5083-5090, 1976.

Hasegawa, A. and Sato, T.: Generation of field-aligned current during substorm, in: Dynamics of the magnetosphere, edited by: Akasofu, S.-I., Reidel, Dordrecht-Holland, p. 529, 1979.

Jones, T. W., Gaalaas, J. B., Ryu, D., and Frank, A.: The MHD Kelvin-Helmholtz instability II: The roles of weak and oblique fields in planar flows, Astrophys. J., 482, 230-244, 1997.

Jones, T. W., Ryu, D., and Frank, A.: 3-d Simulations of the MHD Kelvin-Helmholtz instability, in: Numerical Astrophysics 1998, edited by: Miyama, S., Tomisaka, K. and Hanawa, T., Kluwer, New York, p. 95, 1999.

Keiling, A., Wygant, J. R., Cattell, C., Temerin, M., Mozer, F. S., Kletzing, C. A., Scudder, J., Russell, C. T., Lotko, W., and Streltsov, A. V.: Large Alfvén wave power in the plasma sheet boundary layer during the expansion phase of substorms, Geophys. Res. Lett., 27, 3169-3172, 2000.

Keiling, A., Wygant, J. R., Cattell, C., Johnson, M., Temerin, M., Mozer, F. S., Kletzing, C. A., Scudder, J., and Russell C. T.: Properties of large electric fields in the plasma sheet at 4-7 $R_{E}$ measured with Polar, J. Geophys. Res., 106, 5779-5798, 2001.

Keiling, A., Wygant, J. R., Cattell, C., Peria, W., Parks, G., Temerin, M., Mozer, F. S., Russell, C. T., and Kletzing, C. A.: Correlation of Alfvén wave Poynting flux in the plasma sheet at 4 $7 R_{E}$ with ionospheric electron energy flux, J. Geophys. Res., 107(A7), 1132, doi:10.1029/2001JA900140, 2002.

Keiling, A., Wygant, J. R., Cattell, C. A., Mozer, F. S., and Russell, C. T.: The global morphology of wave Poynting flux: Powering the aurora, Science, 299(5605), 383-386, 2003.

Keiling, A., Parks, G. K., Wygant, J. R., Dombeck, J., Mozer, F. S., Russell, C. T., Streltsov, A. V., and Lotko, W.: Some properties of Alven waves: Observations in the tail lobes and the plasma sheet boundary layer, J. Geophys. Res., 110, A10S11, doi:10.1029/2004JA010907, 2005.

Keller, K. A. and Lysak, R. L.: A two-dimensional simulation of the Kelvin-Helmholtz instability with magnetic shear, J. Geophys. Res., 104, 25097-25103, 1999.

Keller, K. A. and Lysak, R. L.: MHD simulation of magnetospheric waveguide modes, J. Geophys. Res., 106, 8447-8454, 2001.

Keller, K. A., Lysak, R. L., and Song, Y.: A three-dimensional simulation of the Kelvin-Helmholtz instability, in: Magnetospheric Current Systems, edited by: Ohtani, S., Fujii, R.. Hesse, M., and Lysak, R., AGU Monograph 118, American Geophysical Union, Washington, p. 157, 2000.

Kim, J., Hong, S. S., Ryu, D., and Jones, T. W.: Three-dimensional evolution of the Parker instability under a uniform gravity, Ap. J., 506, L139-L142, 1998.

Kim, J., Ryu, D., and Jones, T. W.: Three-dimensional simulations of the Parker instability in a uniformly rotating disk: Ap. J., 557, 464-474, 2001.

Lee, K.-W. and Hau, L.-N.: Characteristics of magnetohydrodynamic waves in Harris-type current sheet with guide magnetic field $B_{y}$, J. Geophys. Res., 113, A12209, doi:10.1029/2008JA013459, 2008.

Lembége, B. and Pellat, R.: Stability of a thick two-dimensional current sheet, Phys. Fluids, 25, 1995-2004, 1982.

Lui, A. T. Y.: Potential plasma instabilities for substorm expansion onsets, Space Sci. Rev., 113, 127-206, 2004.

Lyons, L. R., Zesta, E., Xu, Y., Sánchez, E. R., Samson, J. C., Reeves, G. D., Ruohoniemi, J. M., and Sigwarth, J. B.: Auroral poleward boundary intensifications and tail bursty flows: A manifestation of a large-scale ULF oscillation?, J. Geophys. Res., 107(A11), 1352, doi:10.1029/2001JA000242, 2002.

Lysak, R. L.: The relationship between electrostatic shocks and kinetic Alfvén waves, Geophys. Res. Lett., 25, 2089-2092, 1998.

Lysak, R. L. and Lotko, W.: On the kinetic dispersion relation for shear Alfvén waves, J. Geophys. Res., 101, 5085-5094, 1996.

Lysak, R. L. and Song, Y.: Formation of flux ropes by turbulent reconnection, in: Physics of Magnetic Flux Ropes, edited by: Russell, C. T., Priest, E. R., and Lee, L. C., Geophysical Monograph 58, American Geophysical Union, Washington, D.C., p. 525, 1990.

Lysak, R. L. and Song, Y.: Propagation of Alfvén waves at the plasma sheet boundary layer, in: Proceedings of the 7th International Conference on Substorms, edited by: Pulkinnen, T. and Ganushkina, N., FMI publication, Finnish Meteorological Institute, Helsinki, 2004.

Mende, S. B., Carlson, C. W., Frey, H. U., Peticolas, L. M., and Østgaard, N.: FAST and IMAGE-FUV observations of substorm onset, J. Geophys. Res., 108, 1344, doi:10.1029/2002JA009787, 2003.

Mozer, F. S., Bale, S. D., and Phan, T. D.: Evidence of diffusion regions at a subsolar magnetopause crossing, Phys. Res. Lett., 89, 15002, doi:10.1103/PhysRevLett.89.015002, 2002.

Nakamura, R., Baumjohann, W., Nagai, T., Fujimoto, M., Mukai, T., Klecker, B., Treumann, R., Balogh, A., Rème, H., Sauvaud, J.-A., Kistler, L., Mouikis, C., Owen, C. J., Fazakerley, A. N., Dewhurst, J. P., and Bogdanova, Y.: Flow shear near the boundary of the plasma sheet observed by Cluster and Geotail, J. Geophys. Res., 109, A05204, doi:10.1029/2003JA010174, 2004.

Øieroset, M., Phan, T. D., Fujimoto, M., Lin, R. P., and Lepping, R. P.: In situ detection of reconnection in the Earth's magnetotail, Nature, 412, 414-417, 2001.

O’Neill, S. M., Tregillis, I. L., Jones, T. W., and Ryu, D.: Threedimensional simulations of MHD jet propagation through uniform and stratified external environments, Ap. J., 633, 717-732, 2005. 
Patel, V. L.: Magnetospheric tail as a hydromagnetic waveguide, Phys. Lett., 26A, 596-597, 1968.

Phan, T. D., Drake, J. F., Shay, M. A., Mozer, F. S., and Eastwood, J. P.: Evidence for an elongated (>60 ion skin depths) electron diffusion region during fast magnetic reconnection, Phys. Rev. Lett., 99, 255002, doi:10.1103/PhysRevLett.99.255002, 2007.

Rickard, G. J. and Wright, A. N.: ULF pulsations in a magnetospheric waveguide: comparison of real and simulated satellite data, J. Geophys. Res., 100, 3531-3537, 1995.

Ryu, D. and Jones, T. W.: Numerical magnetohydrodynamics in astrophysics: Algorithm and tests for one-dimensional flow, Astrophys. J., 442, 228-258, 1995.

Ryu, D., Jones, T. W., and Frank, A.: Numerical magnetohydrodynamics in astrophysics: Algorithm and tests for multidimensional flow, Astrophys. J., 452, 785-796, 1995.

Ryu, D., Miniati, F., Jones, T. W., and Frank, A.: A divergence-free upwind code for multidimensional magnetohydrodynamic flows, Astrophys. J., 509, 244-255, 1998.

Ryu, D., Jones, T. W., and Frank, A.: The magnetohydrodynamic Kelvin-Helmholtz instability: A three-dimensional study of nonlinear evolution, Ap. J., 545, 475-493, 2000.

Sato, T. and Iijima, T.: Primary source of large-scale Birkeland currents, Space Sci. Res., 24, 347-366, 1979.

Seboldt, W.: Nonlocal analysis of low-frequency waves in the plasma sheet, J. Geophys. Res., 95, 10471-10479, 1990.

Shay, M. A., Drake, J. F., Rogers, B. N., and Denton, R. E.: Alfvénic collisionless reconnection and the Hall term, J. Geophys. Res., 106, 3759-3772, 2001.

Siscoe, G. L.: Resonant compressional waves in the geomagnetic tail, J. Geophys. Res., 74, 6482-6486, 1969.

Song, Y.: Theoretical constraints on mechanisms for the substorm current wedge, in: Substorms-4, edited by: Kokubun, S. and Kamide, Y., Terra Scientific, Tokyo, p. 543, 1998.

Song, Y. and Lysak, R. L.: Dynamo effect of 3-d time-dependent reconnection in the dayside magnetopause, Geophys. Res. Lett., 16, 911-914, 1989.

Song, Y. and Lysak, R. L.: Alfvénon, driven reconnection and the direct generation of field-aligned current, Geophys. Res. Lett., 21, 1755-1758, 1994.

Song, Y. and Lysak, R. L.: Some theoretical aspects of the solar wind-magnetosphere interaction, Phys. Chem. Earth, 22, 715$721,1997$.
Song, Y. and Lysak, R. L.: Paradigm transition in cosmic plasma physics, magnetic reconnection and the generation of fieldaligned current, in: Magnetospheric Current Systems, edited by: Ohtani, S.-I., Fujii, R., Hesse, M., and Lysak, R. L., AGU Monograph 118, American Geophysical Union, Washington, p. 11, 2000.

Song, Y. and Lysak, R. L.: Towards a new paradigm: From a quasisteady description to a dynamical description of the magnetosphere, Space Sci. Rev., 95, 273-292, 2001.

Sonnerup, B. U. O.: Magnetic field reconnection, in: Solar System Plasma Physics, vol. 3, edited by: Lanzerotti, L. J., Kennel, C. F., and Parker, E. N., North Holland, New York, p. 46, 1979.

Vasyliunas, V. M.: Fundamentals of current description, Magnetospheric currents, edited by: Potemra, T. A., AGU Monograph, Washington, p. 63, 1984.

Volwerk, M., Glassmeier, K.-H., Runov, A., Nakamura, R., Baumjohann, W., Klecker, B., Richter, I., Balogh, A., Rème, H., and Yumoto, H.: Flow burst-induced large-scale plasma sheet oscillation, J. Geophys. Res., 109, A11208, doi:10.1029/2004JA010533, 2004.

Volwerk, M., Glassmeier, K.-H., Nakamura, R., Takada, T., Baumjohann, W., Klecker, B., Rème, H., Zhang, T. L., Lucek, E., and Carr, C. M.: Flow burst-induced Kelvin-Helmholtz waves in the terrestrial magnetotail, Geophys. Res. Lett., 34, L10102, doi:10.1029/2007GL029459, 2007.

Wright, A. N. and Allan, W.: Simulations of Alfvén waves in the geomagnetic tail and their auroral signatures, J. Geophys. Res., 113, A02206, doi:10.1029/2007JA012464, 2008.

Wygant, J. R., Keiling, A., Cattell, C. A., Johnson, M., Lysak, R. L., Temerin, M., Mozer, F. S., Kletzing, C. A., Scudder, J. D., Peterson, W., Russell, C. T., Parks, G., Brittnacher, M., Germany, G., and Spann, J.: Polar comparisons of intense electric fields and Poynting flux hear and within the plasma sheet-lobe boundary to UVI images: An energy source for the aurora, J. Geophys. Res., 105, 18675-18692, 2000.

Wygant, J. R., Keiling, A., Cattell, C. A., Lysak, R. L., Temerin, M., Mozer, F. S., Kletzing, C. A., Scudder, J. D., Streltsov, A. V., Lotko, W., and Russell, C. T.: Evidence for kinetic Alfvén waves and parallel electron energization at $4-6 R_{E}$ altitudes in the plasma sheet boundary layer, J. Geophys. Res., 107(A8), 1201, doi:10.1029/2001JA900113, 2002. 